\title{
Thermal model for small systems
}

\author{
Jean Cleymans ${ }^{1, \star \star}$, Boris Hippolyte ${ }^{2, \star \star \star}$, Helmut Oeschler ${ }^{3, \star \star \star \star}$, Krzysztof Redlich ${ }^{4,5,6, \dagger}$, and \\ Natasha Sharma ${ }^{7}$, \\ ${ }^{1}$ UCT-CERN Research Centre and Department of Physics, \\ University of Cape Town, Rondebosch 7701, South Africa \\ ${ }^{2}$ Institut Pluridisciplinaire Hubert Curien (IPHC), Université de Strasbourg, CNRS-IN2P3, Strasbourg, France \\ ${ }^{3}$ Physikalisches Institut, Ruprecht-Karls-Universität Heidelberg, Heidelberg, Germany \\ ${ }^{4}$ Institute of Theoretical Physics, University of Wrocław, Pl-45204 Wrocław, Poland \\ ${ }^{5}$ ExtreMe Matter Institute EMMI, GSI, D-64291 Darmstadt, Germany \\ ${ }^{6}$ Department of Physics, Duke University, Durham, NC 27708, USA \\ ${ }^{7}$ Department of Physics, Panjab University, Chandigarh 160014, India
}

\begin{abstract}
An analysis is presented of the expectations for particle production in collisions of small nuclei using the thermal model. The maxima observed in particle ratios of strange particles to pions as a function of beam energy in heavy ion collisions, are reduced when considering smaller nuclei. Of particular interest is the $\Lambda / \pi^{+}$ratio which shows a strong maximum surviving even in collisions of small nuclei.
\end{abstract}

\section{Introduction}

A large effort is presently under way to study not only heavy- but also light-ion collisions. This is being motivated by the results obtained in heavy ion collisions like $\mathrm{Pb}-\mathrm{Pb}$ and $\mathrm{Au}-\mathrm{Au}$, for the $\mathrm{K}^{+} / \pi^{+}$, and also other particle ratios. It has been conjectured that these indicate a phase change in nuclear matter [1].

A consistent description of particle production in heavy-ion collisions, up to LHC energies, has emerged during the past two decades using a thermal-statistical model (referred to simply as thermal model in the remainder of this talk). It is based on the creation and subsequent decay of hadronic resonances produced in chemical equilibrium at a unique temperature and baryon chemical potential. According to this picture the hadronic resonances made up of the light flavors are produced in chemical equilibrium.

Indeed some particle ratios exhibit interesting features when studied as a function of the beam energy: (i) a maximum in the $K^{+} / \pi^{+}$ratio, (ii) a maximum in the $\Lambda / \pi$ ratio, (iii) no maximum in the $K^{-} / \pi^{-}$ratio. The maxima occur at a center-of-mass energy of around $10 \mathrm{GeV}$ [2-4]. It is interesting to note that the occurrence of these maxima happens in an energy regime where a maximum baryon

\footnotetext{
$\star \star$ e-mail: jean.cleymasn@uct.ac.za

$\star \star \star$ e-mail: hippolyt@in2p3.fr

$\star \star \star \star$ e-mail: h.oeschler@gsi.de

†e-mail: krzysztof.redlich@ift.uni.wroc.pl

‡e-mail: Natasha.Sharma@cern.ch
} 
density occurs [5] and a transition from baryon-dominated freeze-out to a meson dominated one takes place [4]. An alternative interpretation is that these maxima reflect a phase change [1] to a deconfined state of matter.

The maxima mark a distinction between heavy-ion collisions and p-p collisions as they are not observed in the latter. This shows a clear difference between the two systems which is worthy of investigation.

It is the purpose of the present talk to report on an analysis [6] studying the transition from a small system like a p-p collision to a large system like a $\mathrm{Pb}-\mathrm{Pb}$ or $\mathrm{Au}-\mathrm{Au}$ collision and to follow the genesis of the maxima in certain particle ratios.

\section{The model}

A relativistic heavy-ion collision will go through several stages. At one of the later stages, the system will be dominated by hadronic resonances. The identifying feature of the thermal model is that all the resonances as listed by the Particle Data Group [7] are assumed to be in thermal and chemical equilibrium, an assumption which drastically reduces the number of free parameters and thus this stage is determined by just a few thermodynamic variables namely, the chemical freeze-out temperature $T$, the various chemical potentials $\mu$ determined by the conserved quantum numbers and by the volume $V$ of the system. The latter plays no role when considering ratios of yields. It has been shown that this description is also the correct one [8-11] for a scaling expansion as first discussed by Bjorken [12].

In general, if the number of particles carrying quantum numbers related to a conservation law is small, then the grand-canonical description no longer holds. In such a case conservation of quantum numbers has to be implemented exactly in the canonical ensemble [13,14]. In the case considered here there are two volume parameters: the overall volume of the system $V$, which determines the particle yields at fixed density and the strangeness correlation (cluster) volume $V_{c}$, which reflects the canonical suppression factor and reduces the densities of strange particles. The volume $V_{c}$ is parameterized by the radius $R_{C}$ which serves as a free parameter and defines the range of local strangeness equilibrium.

We start by presenting a brief reminder of the general concepts of the thermal model. In the grand-canonical ensemble, the volume $V$, temperature $T$ and the chemical potentials $\vec{\mu}$ determine the partition function $Z(T, V, \vec{\mu})$. In the hadronic fireball of non-interacting hadrons, $\ln Z$ is the sum of the contributions of all $i$-particle species of energy $E_{i}$ and spin-isospin degeneracy $g_{i}$ by

$$
\frac{1}{V} \ln Z(T, V, \vec{\mu})=\sum_{i} Z_{i}^{1}(T, \vec{\mu}),
$$

where $\vec{\mu}=\left(\mu_{B}, \mu_{S}, \mu_{Q}\right)$ are the chemical potentials related to the conservation of baryon number, strangeness and electric charge, respectively.

The one-particle partition function is given, in Boltzmann approximation, by

$$
Z_{i}^{1} \equiv \frac{g_{i}}{2 \pi^{2}} m_{i}^{2} T K_{2}\left(\frac{m_{i}}{T}\right) \exp (\mu)
$$

The partition function contains all information needed to obtain the number density $n_{i}$ of particle species $i$. Introducing the particle's specific chemical potential $\mu_{i}$, one gets

$$
n_{i}(T, \vec{\mu})=\left.\frac{1}{V} \frac{\partial(T \ln Z)}{\partial \mu_{i}}\right|_{\mu_{i}=0} .
$$


Any resonance that decays into species $i$ contributes to the yields eventually measured. Therefore, the contributions from all heavier hadrons $j$ that decay to hadron $i$ with the branching fraction $\Gamma_{j \rightarrow i}$ are

$$
n_{i}^{\text {decay }}=\sum_{j} \Gamma_{j \rightarrow i} n_{j} .
$$

Consequently, the final yield $N_{i}$ of particle species $i$ is the sum of the thermally produced particles and the decay products of resonances,

$$
N_{i}=\left(n_{i}+n_{i}^{\text {decay }}\right) V .
$$

From (3-5) it is clear that in the grand canonical ensemble the particle yields are determined by the volume of the fireball, its temperature and the chemical potentials.

In general, if the number of particles is small, then the grand-canonical description no longer holds. In such a case conservation laws have to be implemented exactly. We refer here only to strangeness conservation and consider charge and baryon number conservation to be fulfilled on the average. The density of strange particle $i$ carrying strangeness $s$ can be obtained from [13],

$$
\begin{gathered}
n_{i}^{C}=\frac{Z_{i}^{1}}{Z_{S=0}^{C}} \sum_{k=-\infty}^{\infty} \sum_{p=-\infty}^{\infty} a_{3}^{p} a_{2}^{k} a_{1}^{-2 k-3 p-s} \\
I_{k}\left(x_{2}\right) I_{p}\left(x_{3}\right) I_{-2 k-3 p-s}\left(x_{1}\right),
\end{gathered}
$$

where $Z_{S=0}^{C}$ is the canonical partition function

$$
\begin{gathered}
Z_{S=0}^{C}=e^{S_{0}} \sum_{\substack{k=-\infty \\
I_{k}\left(x_{2}\right) I_{p}\left(x_{3}\right) I_{-2 k-3 p}\left(x_{1}\right),}}^{\infty} a_{3}^{p} a_{2}^{k} a_{1}^{-2 k-3 p} \\
\text {. }
\end{gathered}
$$

and $Z_{i}^{1}$ is the one-particle partition function (7) calculated for $\mu_{S}=0$ in the Boltzmann approximation. The arguments of the Bessel functions $I_{s}(x)$ and the parameters $a_{i}$ are introduced as,

$$
a_{s}=\sqrt{S_{s} / S_{-s}}, x_{s}=2 V \sqrt{S_{s} S_{-s}},
$$

where $S_{s}$ is the sum of all $Z_{k}^{1}\left(\mu_{S}=0\right)$ for particle species $k$ carrying strangeness $s$.

In the limit where $x_{n}<1$ (for $n=1,2$ and 3 ) the density of strange particles carrying strangeness $s$ is well approximated by [13]

$$
n_{i}^{C} \simeq n_{i} \frac{I_{s}\left(x_{1}\right)}{I_{0}\left(x_{1}\right)}
$$

From these equations it is clear that in the canonical ensemble the strange particle density depends explicitly on the volume through the arguments of the Bessel functions. This volume might be different from the overall volume $V$ and is denoted as $V_{c}[15,16]$.

To account for the suppression beyond that expected in the canonical ensemble, it was assumed that exact strangeness conservation holds only in a small subvolume $V_{c}$ of a system. The concept of a strangeness correlation volume $V_{c}$ has been used in earlier studies [16-19].

\section{Origin of the maxima}

In the thermal model, the baryon chemical potential decreases continuously with increasing beam energy. At the same time the temperature increases rather quickly until it reaches a plateau. Following 
the rapid rise of the temperature at low beam energies, the $\Lambda / \pi^{+}$and $K^{+} / \pi^{+}$also increase rapidly. This comes to a halt when the temperature reaches its limiting value. However, the baryon chemical potential keeps on decreasing. Consequently, the $\Lambda / \pi$ and $K^{+} / \pi^{+}$ratios follow this decrease due to strangeness conservation as $K^{+}$is produced in associated production together with a $\Lambda$. The two effects combined lead to maxima in both cases. For very high energies, the baryo-chemical potential no longer plays a role $\left(\mu_{B} \approx 0\right)$ and the temperature is constant hence these ratios hardly vary [4].

To show this in more detail we present as an example in figure 1 lines where the $K^{+} / \pi^{+}$and the $\Lambda / \pi^{+}$ratios remain constant in the $T-\mu_{B}$ plane. It should be noted that the maxima of these ratios do not occur in the same position, which remains to be confirmed experimentally. It is also worth noting that the maxima are not on but slightly above the freeze-out curve. The shaded regions indicate the values of these ratios in the $T-\mu_{B}$ plane.
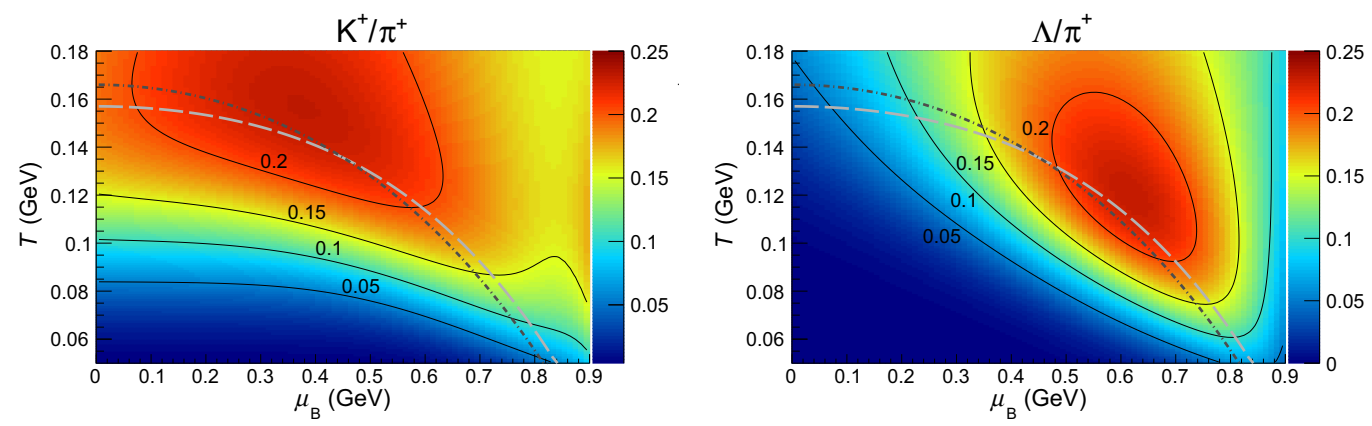

Figure 1. Values of the $K^{+} / \pi^{+}$(left-hand pane) and the $\Lambda / \pi^{+}$(right-hand pane) ratios in the $T-\mu_{B}$ plane. Lines of constant values are indicated. The dashed-dotted line is the freeze-out curve obtained in [3] while the dashed line uses the parameterization given in [21]. Note that the maxima do not occur in the same position.

Similarly, in figure 2 we show in lines where the $\Xi^{-} /\left[\left(\pi^{+}+\pi^{-}\right) / 2\right]$ and the $\Omega^{-} /\left[\left(\pi^{+}+\pi^{-}\right) / 2\right]$ ratios remain constant in the $T-\mu_{B}$ plane. Again, it should be noted that the maxima of these ratios never occur in the same position. Again, it is also worth noting that the maxima are not on but slightly above the freeze-out curve. The shaded regions indicate the values of these ratios in the $T-\mu_{B}$ plane.

The differences in the positions of the maxima are caused by the different masses of the hadrons involved.

\section{Particle ratios for small systems}

To consider the case of the collisions of smaller nuclei we have to take into account the strangeness suppression according to the canonical model, i.e. the concept of strangeness correlation in clusters of a sub-volume $V_{c} \leq V[15,16,20]$.

In the following figures we show the trends of various particle ratios as a function of $\sqrt{s_{\mathrm{NN}}}$. The dependence of $T$ and $\mu_{B}$ on the beam energy is taken from heavy-ion collisions [3]. For p-p collisions slightly different parameters are more suited [22] but were not used. Therefore, the shown calculations give the general trend only. We have ignored the variations of parameters with system size.

We focus on the dependence of the thermal parameters with particular emphasis on the change in the strangeness correlation radius $R_{C}$. The parameters $R=10 \mathrm{fm}$ (which is the value for central $\mathrm{Pb}-\mathrm{Pb}$ collisions) and $\gamma_{S}=1$ are kept fixed. The freeze-out values of $T$ and $\mu_{B}$ will vary with the system size [20], however this has not been taken into account in the present work which aims to give a qualitative description of the effect only. 

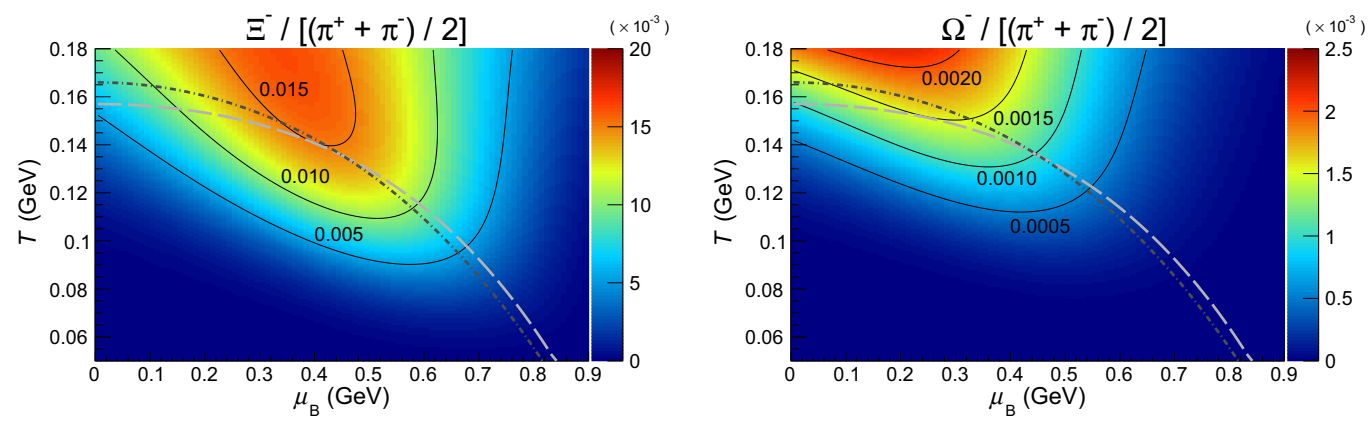

Figure 2. Values of the $\Xi^{-} / \frac{1}{2}\left[\pi^{+}+\pi^{-}\right]$(left-hand pane) and the $\Omega^{-} / \frac{1}{2}\left[\pi^{+}+\pi^{-}\right]$(right-hand pane) ratios in the $T-\mu_{B}$ plane. Lines of constant values are indicated. The dashed-dotted line is the freeze-out curve obtained in [3] while the dashed line uses the parameterization given in [21]. Note that the maxima do not occur in the same position.

The smaller system size is described by decreasing the value of the correlation radius $R_{C}$. This ensures that strangeness conservation is exact in $R_{C}$, and that strangeness production is suppressed with decreasing $R_{C}$.

In figure 3 we show the energy and system size dependence of two particle ratios calculated along the chemical freeze-out line. In figure 3 a maximum is seen in the $K^{+} / \pi^{+}$ratio which gradually disappears when the correlation radius decreases. A different effect is seen in $\Lambda / \pi^{ \pm}$ratio. Here, the gradual decrease of the maximum is also seen but, contrarily to the $\mathrm{K}^{+} / \pi^{+}$ratio, it remains quite prominent even for a small correlation radius. Also, the maximum shifts, for smaller systems, towards higher $\sqrt{s_{\mathrm{NN}}}$. For pp collisions which correspond to a $R_{C}$ of about $1.5 \mathrm{fm}$ [20], they will hardly be observed. It should also be noted that in the thermal model the maxima happen at different beam energies.

In figure 4 we plot the $K^{-} / \pi^{-}$ratio and $\bar{\Lambda} / \pi^{-}$ratios as a function of beam energy for various values of the correlation radius. Due to the different signs of the chemical potentials entering this ratio, as compared to the particle ratios in figure 3 no maximum is obtained for any value of the beam energy or the correlation radius.

Again, we emphasize that the results presented here are of a qualitative nature. In particular there could be changes due to variations with the system size of the temperature and the baryon chemical potential. In addition the strangeness equilibration volume $V_{c}$ could be energy and system size dependent.

The curves in figures 3 and 4 were calculated using THERMUS [23].

The particle ratio $K^{+} / \pi^{+}$of hadrons produced in relativistic nucleus-nucleus collisions has also been studied in the context of the thermal model of hadrons with exact strangeness conservation in [24]. 

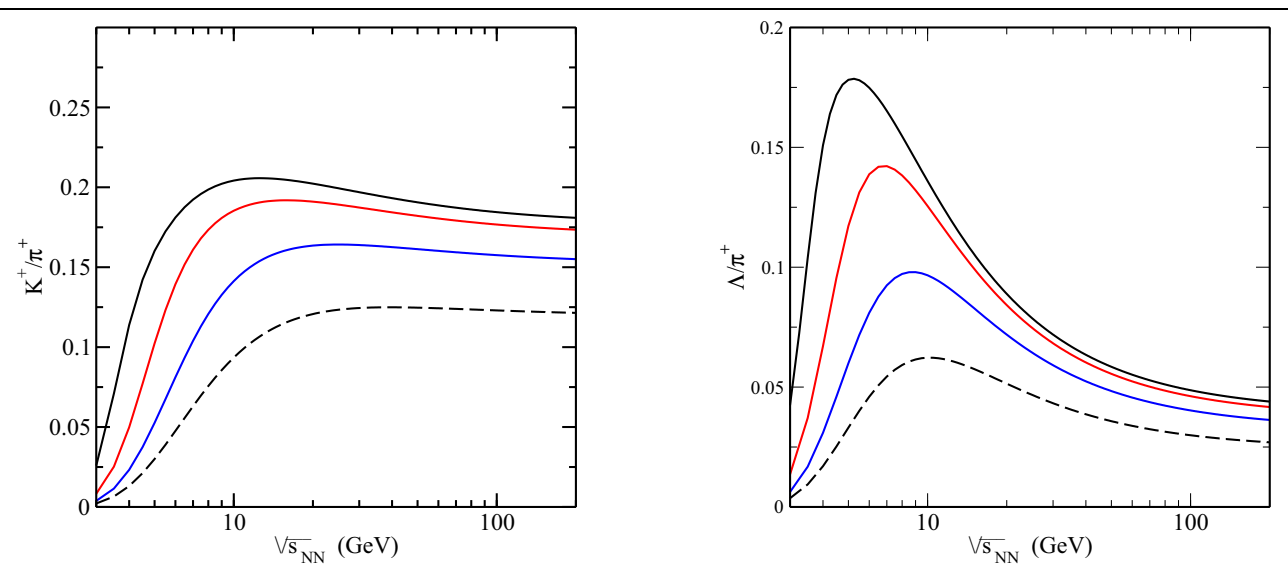

Figure 3. Values of the $K^{+} / \pi^{+}$(left-hand pane) and the $\Lambda / \pi^{+}$(right-hand pane) ratios as a function of invariant beam energy for various strangeness correlation radii $R_{C}$, calculated using the thermal model [23]. The correlation radius is varied from 3.0 (top curve) to 2.5, 2.0, 1.5 and finally $1.0 \mathrm{fm}$ (bottom curve). Note that the $\Lambda / \pi^{+}$ratio is the ratio where the maximum stays most pronounced as the system size is reduced.
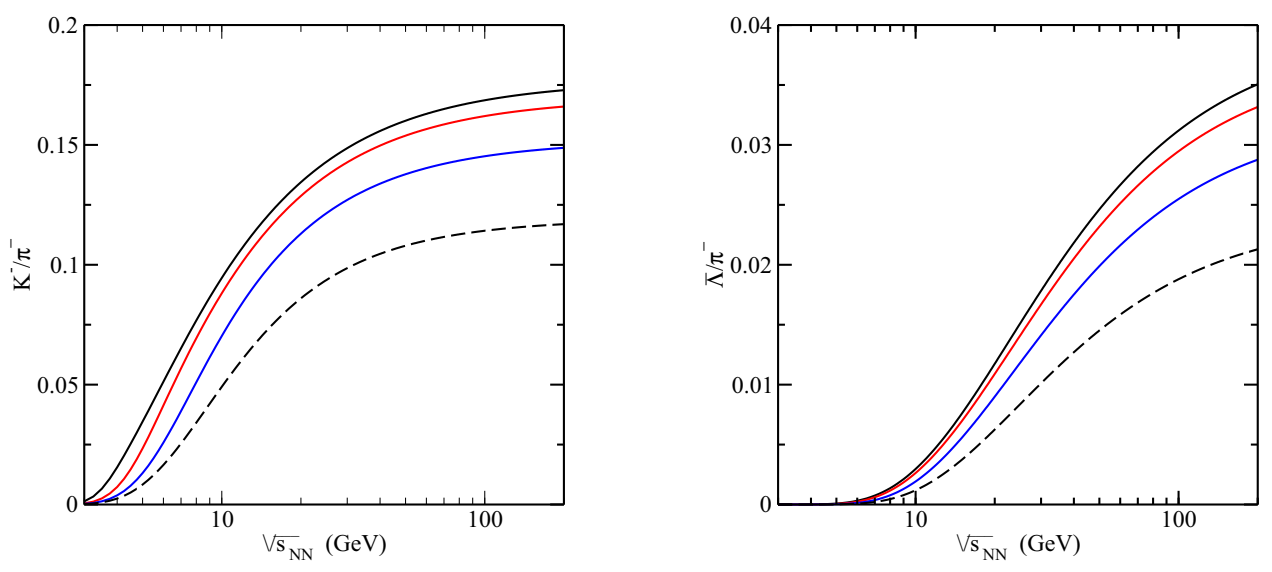

Figure 4. Values of the $K^{-} / \pi^{-}$(left-hand pane) and the $\bar{\Lambda} / \pi^{-}$(right-hand pane) ratios as a function of invariant beam energy for various strangeness correlation radii $R_{C}$, calculated using the thermal model [23]. The correlation radius is varied from 3.0 (top curve) to 2.5, 2.0, 1.5 and finally $1.0 \mathrm{fm}$ (bottom curve).

\section{Conclusions}

The thermal model qualitatively describes the presence of maxima in the $K^{+} / \pi^{+}$and the $\Lambda / \pi^{ \pm}$ratios at a beam energy of $\sqrt{s_{\mathrm{NN}}} \approx 10 \mathrm{GeV}$. In this talk we have described what could possibly happen with different strange particles and pion yields in collisions of smaller systems due to constraints imposed by exact strangeness conservation. In particular, the $\Lambda / \pi^{+}$ratio still shows a clear maximum even small systems. The pattern of these maxima is also quite special as they are not always at the same beam energy. 


\section{Acknowledgments}

K.R. acknowledges the supported by the National Science Center, Poland under grant Maestro, DEC2013/10/A/ST2/00106. N.S. acknowledges the support of DST-SERB Ramanujan Fellowship (D.O. No. SB/S2/RJN- 084/2015).

\section{References}

[1] M. Gazdzicki and M.I. Gorenstein, Acta Phys. Polon. B 30, 2705 (1999)

[2] A. Andronic, P. Braun-Munzinger and J. Stachel, Nucl. Phys. A 772, 167 (2006)

[3] J. Cleymans, H. Oeschler, K. Redlich, S. Wheaton, Phys. Rev. C 73, 034905 (2006)

[4] J. Cleymans, H. Oeschler, K. Redlich, S. Wheaton, Phys. Lett. B 615, 50 (2005)

[5] J. Randrup and J. Cleymans Eur. Phys. J. A 52, 218 (2016)

[6] J. Cleymans, B. Hippolyte, H. Oeschler, K. Redlich, N. Sharma, arXiv:1603.0953[hep-ph]

[7] K.A. Olive et al., Particle Data Group, Chin. Phys. C 38, 090001 (2014)

[8] J. Cleymans, Proceedings, 3rd International Conference on Physics andastrophysics of quarkgluon plasma (ICPA-QGP '97, 1998) 55

[9] J. Cleymans and K. Redlich, Phys. Rev. C 60, 054908 (1999)

[10] S.V. Akkelin, P. Braun-Munzinger and Yu. M. Sinyukov, Nucl. Phys. A 710, 439 (2002)

[11] W. Broniowski and W. Florkowski, Phys. Rev. Lett. 87, 272302 (2001)

[12] J.D. Bjorken, Phys. Rev. D 27, 140 (1983)

[13] P. Braun-Munzinger, J. Cleymans, H. Oeschler, K. Redlich, Nucl. Phys. A 697, 902 (2002)

[14] P. Braun-Munzinger, K. Redlich, J. Stachel, in Quark Gluon Plasma 3, eds. R.C. Hwa and XinNian Wang (World Scientific Publishing, 2003)

[15] J. Cleymans, H. Oeschler, K. Redlich, Phys. Rev. C 59, 1663 (1999)

[16] S. Hamieh, K. Redlich, A. Tounsi, Phys. Lett. B 486, 61 (2000)

[17] A. Tounsi, A. Mischke, K. Redlich, Nucl. Phys. A 715, 565 (2003)

[18] H. Caines, J. Phys. G 32, S171 (2006)

[19] C. Höhne, F. Pühlhofer, R. Stock, Phys. Lett. B 640, 96 (2006)

[20] I. Kraus, J. Cleymans, H. Oeschler, K. Redlich, S. Wheaton, Phys. Rev. C 76, 064903 (2007)

[21] V. Vovchenko, V.V. Begun, M.I. Gorenstein, Phys. Rev. C 93, 064906 (2016)

[22] J. Cleymans, S. Kabana, I. Kraus, H. Oeschler, K. Redlich, N. Sharma, Phys. Rev. C 84, 054916 (2011)

[23] S. Wheaton, J. Cleymans, M. Hauer, Comp. Phys. Comm. 180, 84-106 (2009)

[24] V.D. Toneev and A.S. Parvan, J. Phys. G. 31, 583 (2005) 